\title{
Investigation of slip transmission behavior across grain boundaries in polycrystalline $\mathrm{Ni}_{3} \mathrm{Al}$ using nanoindentation
}

\author{
P.C. Wo and A.H.W. Ngan ${ }^{\text {a) }}$ \\ Department of Mechanical Engineering, The University of Hong Kong, \\ Hong Kong, People's Republic of China
}

(Received 10 June 2003; accepted 25 September 2003)

\begin{abstract}
The influence of grain boundaries on material deformation in $\mathrm{Ni}_{3} \mathrm{Al}$ was investigated by relating the material pile-up at grain boundaries and the propagation of slip across grain boundaries to the misorientation between the corresponding grains. Indentation tests were carried out using micro- and nanoindentation at distances shorter than the radius of indent size from a grain boundary on $\mathrm{Ni}_{3} \mathrm{Al}$. The indents were observed using scanning electron microscopy and non-contact-mode atomic force microscopy. Repeated experimentation did not reveal a rising trend of hardness near grain boundaries, indicating that hardness is not a sensitive parameter to measure grain boundary strengthening effects. However, it was observed that the slip transfer behavior across a grain boundary has a strong dependence on a local misorientation factor $m^{\prime}$ relating the misorientation of slip planes and slip directions on either side of the grain boundary. This result agrees with the fundamental assumption in the physical explanation of the Hall-Petch effect.
\end{abstract}

\section{INTRODUCTION}

Subgranular microhardness indentation has frequently been carried out to measure the so-called degree of hardening of grain boundaries in bicrystals or large-grained polycrystalline specimens. ${ }^{1-6}$ Grain boundary hardening has traditionally been attributed to grain boundary segregation of impurity atoms, as in the case of doped zonerefined metals such as $\mathrm{Pb}, \mathrm{Sn}$, and $\mathrm{Zn},{ }^{1}$ tin segregation in alpha iron-tin alloy, ${ }^{2}$ calcium segregation in $\mathrm{NaCl}$ bicrystals, ${ }^{3}$ and impurity segregation in niobium bicrystals. $^{4,5}$ On the other hand, it may be tempting to relate grain boundary hardening observed by microindentation to the difficulty in slip transmission across grain boundaries. By conducting macroscopic tensile tests on highpurity aluminium, Wyrzykowski and Grabski ${ }^{6}$ concluded that the Hall-Petch slope $k_{y}$ depends on the distribution function of the grain boundary diffusivity, indicating a dependence of $k_{y}$ on the grain boundary structure. By analyzing some of the grain boundary hardening results in the literature, Ngan and $\mathrm{Chiu}^{7}$ observed that, even in cases where the hardening effect was proposed to be due to impurity segregation, the hardness seems to obey an analogous Hall-Petch form in which the hardness increases linearly with the inverse of the square root of the distance between the indent and the grain boundary.

\footnotetext{
a) Address all correspondence to this author. e-mail: hwngan@hkucc.hku.hk
}

Ngan and Chiu argued that this is a supporting evidence for the slip transmission hypothesis. One material example in which slip transmission may be of special relevance is $\mathrm{Ni}_{3} \mathrm{Al}$. In this material, earlier tensile or compression test results have shown that the Hall-Petch slope $k_{y}$ is significantly reduced upon boron doping in the nickel-rich regime. ${ }^{8}$ Recently, Lee et al. ${ }^{9}$ have performed microhardness tests on boron-free and boron-doped $\mathrm{Ni}_{3} \mathrm{Al}$ bicrystals and have reported that grain boundary hardening as measured by subgranular microindentation is remarkably decreased by boron doping, indicating a potential link between the degree of grain boundary hardening and the Hall-Petch slope $k_{y}$.

To clarify the role of grain boundary slip transmission, we have performed subgranular indentation in $\mathrm{Ni}_{3} \mathrm{Al}$. In most of the previous investigations, ${ }^{1-5,9}$ only the measured hardness values were reported, but no microscopic information about the shape of the indents was given. Because hardness is defined as the indentation load divided by the projected indent area, at constant load, an observed hardening effect means an observed reduction in indent size. However, if in a given material a grainboundary hardening effect is due to difficulty in slip transmission across grain boundaries, an indent situated nearer to a grain boundary should not have its size reduced self-similarly - the indent shape should indeed be asymmetrical with greater reduction in dimensions on the sides facing the grain boundary than those away from the grain boundary. In other words, if a grain boundary is to 
produce an observed hardening effect, the indent shape should be distorted by the grain boundary. It is likely that such topographical information might provide useful information about the slip transmission, but so far no systematic investigation on this has been conducted. We select $\mathrm{Ni}_{3} \mathrm{Al}$ to investigate this effect because in the undoped state, the grain boundaries in $\mathrm{Ni}_{3} \mathrm{Al}$ may offer a high resistance to intergranular slip transmission, ${ }^{8,9}$ and hence the unsymmetrical indent phenomenon, if present, should be very prominent in this material.

\section{EXPERIMENTAL}

An ingot with elemental composition of 74 at.\% $\mathrm{Ni}$ and 26 at.\% $\mathrm{Al}$ was prepared by melting pure $\mathrm{Ni}$ $(>99.92 \%)$ and $\mathrm{Al}(>99.79 \%)$ in an induction furnace. It was homogenized at $1100{ }^{\circ} \mathrm{C}$ in a vacuum better than $10^{-5}$ mbar for more than $74 \mathrm{~h}$ before furnace cooling to room temperature, resulting in elongated grains with an average width of $180 \mu \mathrm{m}$ and length about $500 \mu \mathrm{m}$. A sample was cut into dimensions of $10 \times 7 \times 5 \mathrm{~mm}$ and then mechanically polished to $1 \mu \mathrm{m}$ followed by electropolishing in an electrolyte containing 10\% perchloric acid in methanol. A transmission electron microscope sample was made and was examined for the initial condition of the indentation samples. To prepare the transmission electron microscopy (TEM) samples, thin disks of 3-mm diameter were cut from the ingot and were mechanically ground to $1 \mu \mathrm{m}$ followed by twin-jet polishing in the same electrolyte as that used for the bulk samples.

Three types of indentation tests were carried out. The first type was carried out with a Buehler Micromet 2100 (Lake Bluff, IL) series microhardness indenter with a Vickers tip. The second type was performed with a Hysitron Triboscope (Minneapolis, MN) nanoindentation transducer on a Park Scientific Instruments AutoProbe CP (Sunnyvale, CA) scanning platform. The loading profile used was either a simple schedule consisting of a linear load ramp followed by linear unloading or an exponential loading schedule superimposed with sinusoidal modulations at regular intervals. The simple load-unload schedule is often used in the literature ${ }^{1-5,9}$ and is used here for purposes of comparison. The exponential loading process is meant to give a more consistent measurement of hardness for two reasons. First, the exponentially increasing load tends to yield load-independent hardness ${ }^{10}$ and second, using force modulation to measure the tip-sample contact stiffness as opposed to the OliverPharr unloading method would minimize the influence of thermal drift on the hardness measurement. ${ }^{11}$ Indentations were made with a Berkovich tip at different distances from selected grain boundaries to investigate any grain boundary effect on hardness at various distances from the grain boundaries.
The third type of indentation tests were performed with a CSM Instruments SA nanoindenter (Neuchâtel, Switzerland) using a simple load-unload profile with maximum force of $300 \mathrm{mN}$. Both cube-corner and Berkovich indenters were used for making indents along selected grain boundaries on the $\mathrm{Ni}_{3} \mathrm{Al}$ sample. The impressions were examined qualitatively by scanning electron microscopy (SEM) backscattered imaging using either a Cambridge Stereoscan 360 or a 440 microscope (LEO Electron Microscopy Ltd., Cambridge, U.K.). Topographic profiles around the indents were quantitatively measured using non-contact-mode atomic force microscopy (AFM) on a Park Scientific Instruments AutoProbe $\mathrm{CP}$ scanning platform.

\section{RESULTS}

\section{A. General considerations}

TEM examinations show that the initial dislocation density in the virgin sample is very low. In repeated observations, not a single dislocation could be found within a region larger than $20 \times 20 \mu \mathrm{m}$. Considering that the indents made using the largest load of $300 \mathrm{mN}$ were much smaller than this dimension, the indentations in this work, especially during the initial stage, were likely made on dislocation-free locations.

Figures 1(a)-1(c) shows the hardness values measured at various distances from different selected grain boundaries on the $\mathrm{Ni}_{3} \mathrm{Al}$ sample. The distance here refers to that between the indent center and the nearest grain boundary portion within the grain. The samples used were polycrystalline, and to avoid the influence of any potential grain boundaries that might exist beneath the surface of the sample, grains with large cross-sections were selected for the indentation tests. The large grain cross sections exposed on the sample surface are probably the diameter planes of the grain, so that any subsurface grain boundaries should be at a sufficiently large depth compared to the indent depth and should not interfere with the indent. The distance scale in the abscissa of Fig. 1 represents the shortest distance of an indent from the nearest grain boundary portion. This distance in fact becomes ill defined if it is larger than the radius of curvature of the selected grain boundary portion. The radius of curvature of the grains is of the order $100 \mu \mathrm{m}$, and a fraction of this, say $50 \mu \mathrm{m}$, could serve as an upper bound for the distance scale. On the other hand, the plasticity zone around an indent is unlikely to be larger than a few times the indent radius. Samuels and Mulhearn ${ }^{12}$ have suggested that the plastic boundary for diamond pyramid indenters lies at around 2.7 times the diameter of the indent. A similar conclusion can be drawn from the well known "cavity model" by Johnson. ${ }^{13}$ Hence, the grain boundary is expected to lose its influence if the distance of the indent from it is larger than a few times 


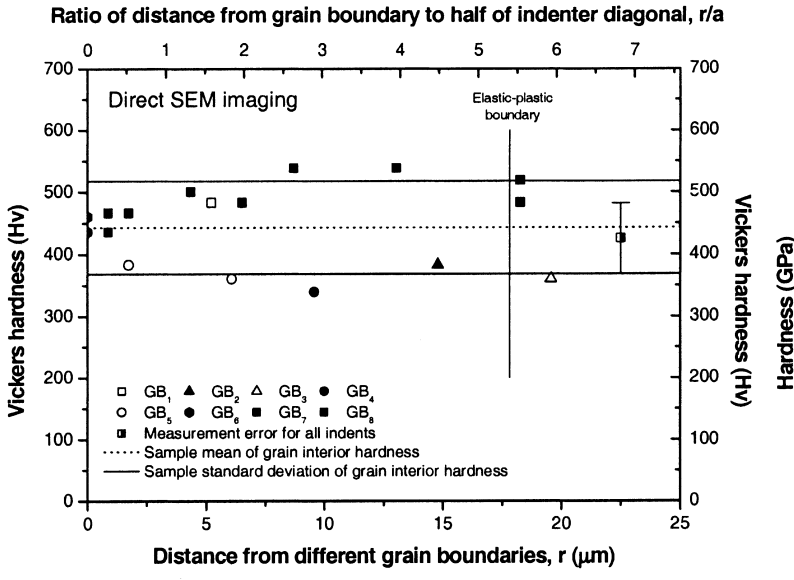

(a)

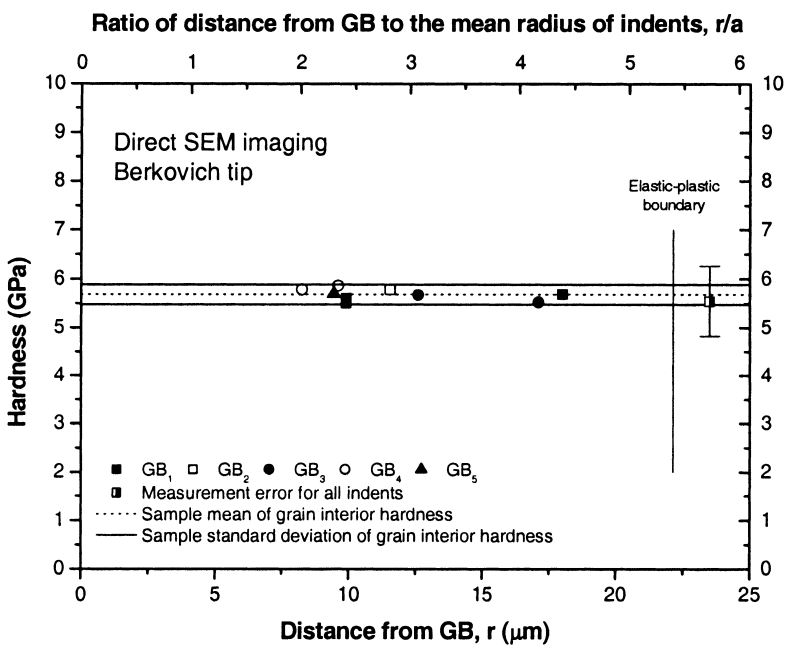

(b)(ii)
Ratio of distance from GB to the mean radius of indents, r/a

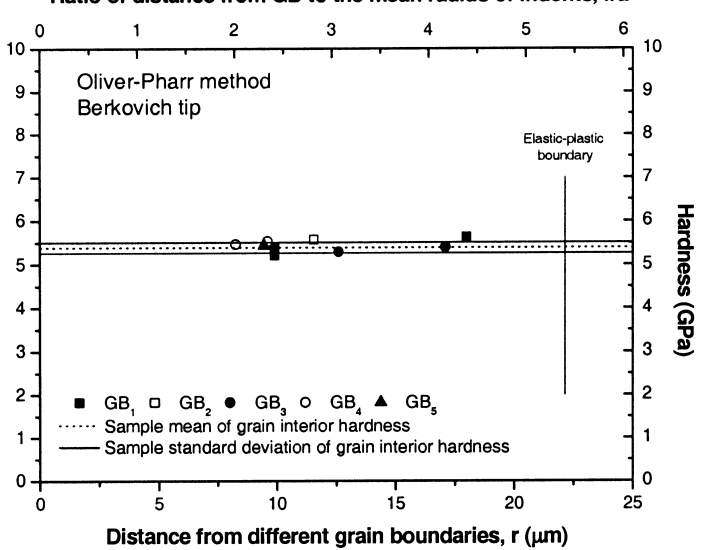

(b)(i)

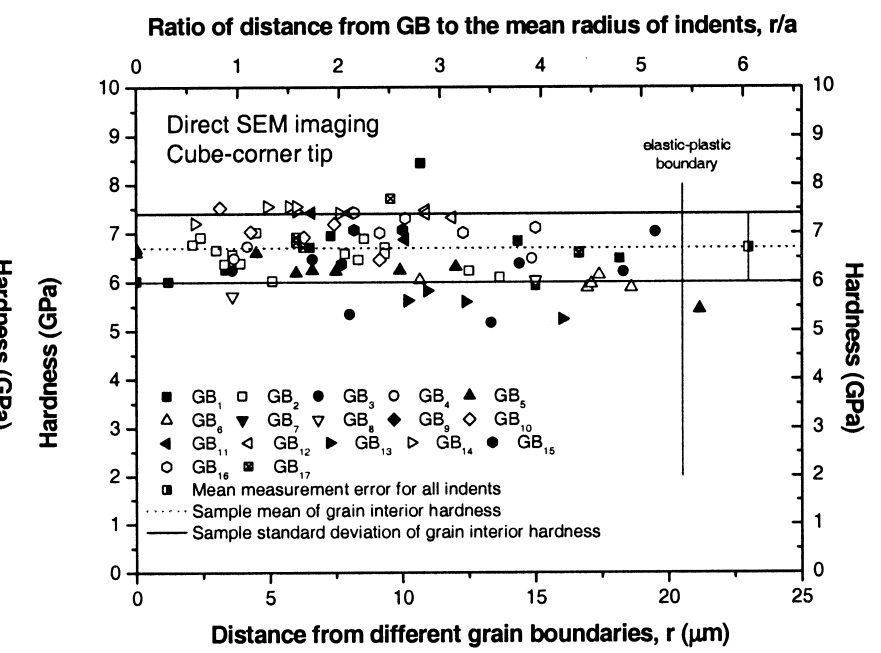

(c)

FIG. 1. Hardness $H$ against the shortest distances from an indent to the nearest grain boundary in the $\mathrm{Ni}_{3} \mathrm{Al}$ sample. The $H$ values were obtained by indentation using (a) a Vickers tip and a load of $10 \mathrm{gf}$, (b) a Berkovich tip and a maximum load of $300 \mathrm{mN}$, and (c) a cube-corner tip and a maximum load of $300 \mathrm{mN}$. The $H$ values shown in (a), (b, ii), and (c) were obtained by direct SEM imaging, whereas those in (b, i) were obtained by the Oliver-Pharr method. The mean $H$ value for the grain interior is indicated by the dotted line while the horizontal solid lines show the upper and lower bounds of one standard deviation from such mean value. The data points show the $H$ value at distances less than a few times the indent size. The error bar on the right of the diagrams shows the variations due to judgmental errors of the indent size.

the indent radius. The top abscissa scale in Fig. 1 shows the distance as multiples of the indent radius $a$, and the data points in Fig. 1 are those such that the distance from the grain boundary is within a few times $a$. The indent radius $a$ here is defined as the radius of the circle that has the same area as the indent itself (Fig. 2). For those indents that are further than about ten times the indent radius from any grain boundary, they are labeled as indents made in the grain interior. More than 10 indents were made in the grain interior in each set of tests, and the average hardness value obtained is indicated in Fig. 1 as a dotted horizontal line. Also shown as solid horizontal lines in Fig. 1 are the estimated variations of the grain interior hardness, which are one standard deviation on either side of the mean value.

The hardness values as shown in Figs. 1(b) and 1(c) are calculated according to the definition of indentation load divided by the projected area of the indent. The Vickers hardness in Fig. 1(a) is defined as load divided by the sloping area of the indentation, and it should therefore be 0.927 times the hardness based on projected indent area. ${ }^{14}$ The indentation load used in the hardness calculation in Figs. 1(b) and 1(c) was the value detected by the nanoindenter, which was usually deviated within $\pm 0.5 \mathrm{mN}$ from the preset value. The projected indent areas in Figs. 1(a), 1(b, ii), and 1(c) were measured from the postindentation SEM images of the indents; examples of these are shown in Fig. 2 for a few indents made at $300 \mathrm{mN}$. The major source of error in the measurement of the indent area this way comes from the judgmental errors on the boundaries of the indent. Typical estimates of these errors are also shown in Fig. 2, as an illustration. 

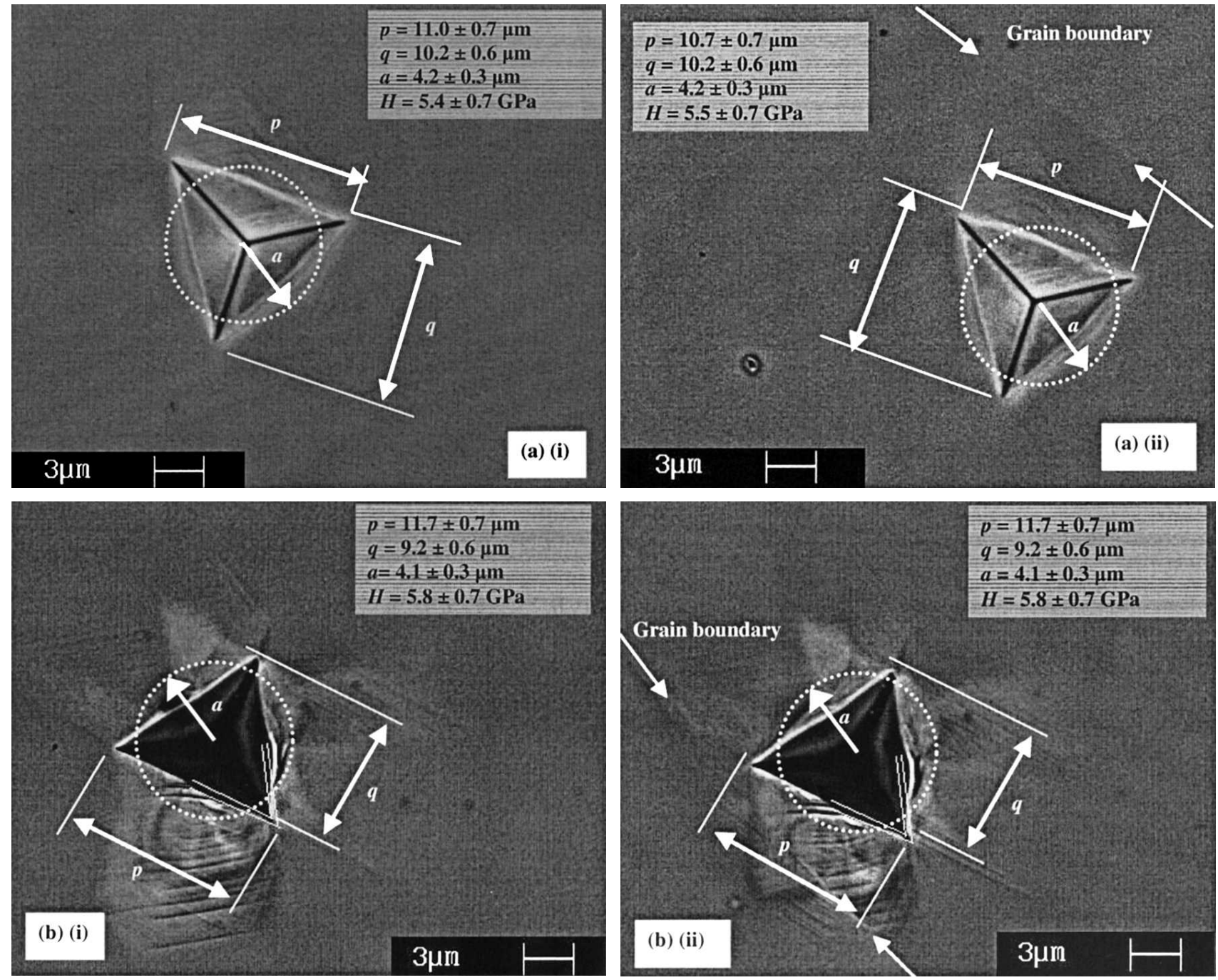

FIG. 2. SEM backscattered images showing typical (a) Berkovich and (b) cube-corner indents made with $300 \mathrm{mN}$ maximum applied load (a, i), $(b, i)$ in the grain interior and (a, ii), (b, ii) near a grain boundary. Measurements of the indent sizes are indicated together with the estimated error due to judgmental error on the indent boundaries, as indicated by the lines at the corners of the indents in (b). The circle superimposed on the indents has the same area as the projected area of the indent.

Taking the measurements and the corresponding error estimated in Fig. 2(a, i) as an example, the projected indent area is $A=\pi a^{2}=3.14(4.2 \pm 0.3)^{2}=55.4 \pm$ $7.9 \mu^{2}$. With an average measurement error of the projected indent area of $\pm 7.9 \mu \mathrm{m}^{2}$, the measurement error of the hardness value $H$ is about $\pm 0.7 \mathrm{GPa}$ (or $\pm \mathrm{HV} 70$ ) at $300 \mathrm{mN}$, and this is also indicated in Fig. 2. The error bars near the right axes of Figs. 1(a), 1(b, ii), and 1(c) indicate this judgmental error. It can be seen that the standard deviations of the grain interior hardness measured from the sample of the Vickers indents in Fig. 1(a) and the sample of the cube-corner indents in Fig. 1(c) are quite close to the judgmental errors, but the standard deviation from the sample of the Berkovich indents in Fig. 1(b, ii) is significantly smaller than the judgmental error. This is probably because of the relatively smaller sample size of six samples used in the Berkovich, graininterior indents. The grain-interior sample sizes for the Vickers and cube-corner indents in Figs. 1(a) and 1(c) were 20 and 31 , respectively.

To investigate the effect of the orientation of the indent about the indentation axis, two types of indents with two different orientations denoted as $R_{\mathrm{A}}$ and $R_{\mathrm{B}}$ were made using a cube-corner indenter under a $300 \mathrm{mN}$ load on the same grain as shown in Fig. 3. Here, the indentation axis is parallel to the [ $\overline{67} 5641]$ orientation of the crystal, and one edge of the $R_{\mathrm{A}}$-oriented indents [Fig. 3(a)] is parallel to the [72 53 45] direction. The $R_{\mathrm{B}}$ indents [Fig. 3(b)] are roughly $26^{\circ}$ clockwise relative to $R_{\mathrm{A}}$. Magnified SEM images of typical $R_{\mathrm{A}}$ and $R_{\mathrm{B}}$ indents shown in Figs. 3(a) and $3(\mathrm{~b})$, respectively, show that material appears to pile up at the edges of both indents, but to a different extent 


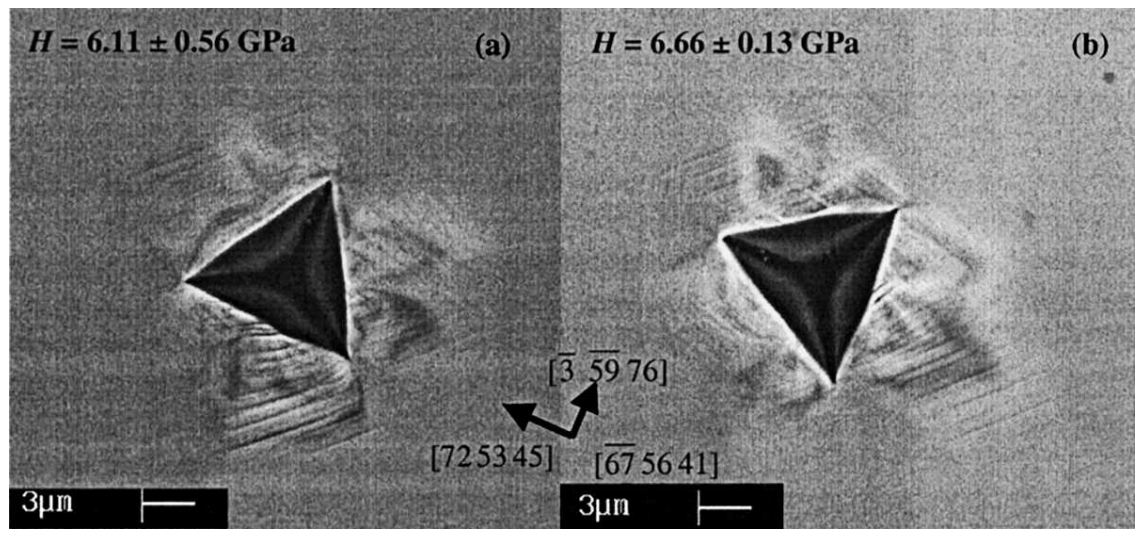

FIG. 3. SEM images showing the (a) $R_{\mathrm{A}}$ and (b) $R_{\mathrm{B}}$ oriented cube-corner indents made with $300 \mathrm{mN}$ load on a grain. Magnified SEM images of typical indents with orientations $R_{\mathrm{A}}$ and $R_{\mathrm{B}}$ show similar sizes of slip zones and same sets of slip traces around the indents.

at each of the edges. Also, slip traces appearing as straight lines can be seen around the indents. The same sets of parallel slip traces can be seen to occur in both indents, indicating that the same active systems were involved in both cases. Although the detailed geometries of the slip zones around the two indents are different as can be expected, their sizes are, however, very similar. The hardness values for eight $R_{\mathrm{A}}$-oriented indents studied have a mean value of $6.11 \mathrm{GPa}$ and a standard deviation of $0.56 \mathrm{GPa}$. The mean hardness value for the five $R_{\mathrm{B}}$-oriented indents studied is $6.66 \mathrm{GPa}$ with a standard deviation of $0.13 \mathrm{GPa}$. The differences amongst these $H$ values are compatible with the expected error of about $0.58 \mathrm{GPa}$ in the projected indent area measurements. These results suggest that the effect of indent rotation on the $H$ value within the same grain is not significant, in agreement with the conclusion drawn by Wang et al. ${ }^{15}$

\section{B. Microhardness results}

Figure 1(a) shows the results of an experiment similar to that carried out by Lee et al. ${ }^{9}$ on boron-free $\mathrm{Ni}_{3} \mathrm{Al}$ in which a Vickers tip was used with 10 gf load. Here, several grain boundaries were selected for the test, and the Vickers hardness obtained is plotted against the distance from the corresponding grain boundaries. A word of caution about the hardness value quoted here is necessary. Our Buehler Micromet hardness tester is a traditional type of indenter, in which the indent size is measured by a digital filar micrometer when viewed under an optical microscope. Such a measurement method gives $d=7.90 \pm 0.95 \mu \mathrm{m}$ (average value) at a load of $10 \mathrm{gf}$, where $d$ is the diagonal length of a Vickers indent, and the error of $\pm 0.95 \mu \mathrm{m}$ here is our best estimate of the judgmental error involved in determining the end points of the indent diagonals when viewed using the optical microscope of the indenter. With this judgmental error, the hardness calculated from the formula $H V=1.854 \times W /$ $d^{2}$ is HV $297.0 \pm 71.5$. However, the measurement of lengths in the range $0-30 \mu \mathrm{m}$ by the filar micrometer of our indenter was found to be erroneous upon verification with SEM imaging. By comparing with SEM imaging, the filar micrometer of our microindenter was found to give consistent overestimation of lengths shorter than about $30 \mu \mathrm{m}$. When viewed using SEM, the indents produced by a $10 \mathrm{gf}$ load by our microindenter were only about $6.60 \pm 0.44 \mu \mathrm{m}$ compared to the $7.90 \pm 0.95 \mu \mathrm{m}$ obtained directly by the filar micrometer of the indenter. Hence, instead of using the filar micrometer in the microindenter, all indents were imaged by SEM and the hardness values were calculated from the sizes of the indents as seen in the electron micrographs. These hardness values are shown in Fig. 1(a). As indicated in the figure, the microhardness of the grain interior varies about a mean value of HV 443.0, with a standard deviation of HV 74.6. The hardness values obtained near the different grain boundaries lie between HV 339.4 and HV 538.3. It can therefore be concluded that the hardness values near the selected grain boundaries are not statistically different from those measured in the grain interior.

The observations here are very different from those made by Lee et al. ${ }^{9}$ First, the hardness range they observed for a boron-free $\mathrm{Ni}_{3} \mathrm{Al}$ bicrystal was from $\mathrm{HV} 190$ to 235 , versus our values from HV 339.4 to 538.3. It should be noted that the same load of $10 \mathrm{gf}$ is used in both cases and so the difference should not be due to the well-known indentation size effect. ${ }^{16}$ Second, the error reported by Lee et al. was only around $\pm \mathrm{HV} 3$, which is much smaller than the statistical variation of about $\pm \mathrm{HV}$ 75 observed in our results in Fig. 1(a). We notice from the report of Lee et al. ${ }^{9}$ that they used a similar digital microhardness tester to ours, but they did not use SEM or any other means to verify the measured indent sizes. Furthermore, they reported a measurement error of only $\pm 0.1 \mu \mathrm{m}$ for the indent size, which we reckon as impossibly small in view of the resolution limit of any optical microscope. From our repeated experimentation, we find it unconvincing to conclude an error as small as $\pm \mathrm{HV} 3$ 
for the hardness measurement of hard materials like $\mathrm{Ni}_{3} \mathrm{Al}$ using a digital microhardness tester similar to the one used in the current study.

\section{Depth-sensing indentation results}

Both a Berkovich and a cube-corner tip were used to perform indentation tests using our CSM Instruments SA nanoindenter at various distances from selected grain boundaries, and the results are shown in Figs. 1(b) and 1(c), respectively. For the results from the Berkovich tip, two different methods, namely, the Oliver-Pharr $\operatorname{method}^{17}$ and direct imaging by SEM, were used to estimate the sizes of the indentations. In the Oliver-Pharr method, the tip-sample contact depth is given by

$$
h_{\mathrm{c}}=h-\epsilon \frac{P}{S},
$$

here $P$ is the maximum load, $h$ is the maximum indenter displacement, $S$ is the contact stiffness at unload, and $\epsilon=0.72$. Once the contact depth $h_{\mathrm{c}}$ is known, the contact area can be calculated using the precalibrated area function of the tip. The hardness results given in Fig. 1(b, i) were obtained by the Oliver-Pharr method whereas those shown in Fig. 1(b, ii) were obtained by direct SEM imaging.

\section{Results with a Berkovich indenter}

Comparing Figs. 1(b, i) and 1(b, ii), the mean $H$ value in the grain interior obtained by the Oliver-Pharr method is about $0.3 \mathrm{GPa}$ lower than that evaluated by direct SEM imaging. A similar observation can be made for the hardness values near a grain boundary (from about 6 to $24 \mu \mathrm{m}$ from a grain boundary). The slight underestimation of the hardness by the Oliver-Pharr method is due to its overestimation of the contact depth $h_{\mathrm{c}}$ in Eq. (1), and this is likely to be due to indentation creep effects during unload which, if not corrected, would lead to overestimation of the unloading stiffness $S$ and hence $h_{\mathrm{c}}{ }^{18-20}$ However, because the discrepancy here is only about $0.3 \mathrm{GPa}$, which is $5 \%$ of the mean hardness value, we reckoned that the results are acceptable and did not take the extra steps to do creep correction.

It can be seen from Fig. 1(b, ii) that some hardness values near the grain boundaries are about $0.3 \mathrm{GPa}$ higher than the mean $H$ measured in the grain interior. It is important to determine the statistical significance of such a difference before we can accept or rule out the existence of a grain boundary effect on the measured hardness. To do this, the $H$ data are regarded as belonging to two groups. One group consists of data at distances shorter than about 5.5 times the indent radius and is treated as data representing a typical grain boundary region. This group has a sample size of nine. The second group consists of data for distances greater than 5.5 times the indent radius and is regarded as data representing the grain interior, the sample size of which is six. By using standard hypothesis testing methods assuming the Student $t$ distribution for the sample means, it was found that at a significance level of 5\%, the hardness values near the grain boundaries are not significantly higher than those within the grain interior. Therefore, one has to conclude from the results here that a grain boundary effect on the measured hardness has not been observed.

The above conclusion is further supported by SEM imaging of the indent shapes near the grain boundaries. Figure 2(a) shows the SEM images of a typical indent made near a grain boundary and one made within the grain interior under the same load of $300 \mathrm{mN}$. It can be seen that the size and shape of the indents are the same. Many other images near the grain boundaries and within the grain interior were compared this way, and the result is the same. One is therefore compelled to conclude that in our $\mathrm{Ni}_{3} \mathrm{Al}$ sample, the measured hardness is not affected by the presence of a grain boundary in the neighborhood of the indentation.

\section{Results with a cube-corner indenter}

Figure 1(c) shows the hardness results obtained by a cube-corner indenter with a $300 \mathrm{mN}$ load on different grains and at various distances from some grain boundaries. The hardness values here are all calculated based on direct SEM imaging of the indents. Similar to the results obtained by the Berkovich indenter above, some hardness values in a grain boundary region are higher (by up to $1 \mathrm{GPa}$ ) compared to the mean value for the grain interior. Hypothesis testing using sample sizes of 31 for the grain interior and 87 for the grain boundary region again showed that the hardness measured within the grain boundary region is not significantly higher than that observed in the grain interior. This conclusion is again confirmed by SEM imaging, which shows that the typical indents made close to and far away from a grain boundary on the same grain and under the same load have identical shape and dimensions [Fig. 2(b)].

The indentation results also show that the hardness measured using a Berkovich indenter is about $1 \mathrm{GPa}$ lower than that measured with a cube-corner indenter under the same load. This result is verified by the slightly larger projected areas of the Berkovich indents [Fig. 2(a)] compared to those of the cube-corner indents [Fig. 2(b)] under the same load.

Careful inspection of Figs. 1(b, ii) and 1(c) shows that the variation of hardness measured on a fixed grain (with reference to a fixed grain boundary) is relatively small, with a maximum deviation of $\pm 0.4 \mathrm{GPa}$. However, it became quite obvious that the hardness values measured on different grains vary from about $6.0 \mathrm{GPa}$ near two grain boundaries labeled $\mathrm{GB}_{3}$ and $\mathrm{GB}_{6}$ to approximately 7.5 GPa near another labeled $\mathrm{GB}_{14}$. These results suggest 
that the $H$ value exhibits a strong dependence on the crystallographic orientation of the sample.

Apart from the observation that the measured hardness does not show any statistically significant difference near a grain boundary or within a grain, the loaddisplacement curves of these indentations also do not exhibit any remarkable difference. Figure 4 shows the load-displacement curves of some typical cube-corner indentations on the grain interior, near a grain boundary, and right on a grain boundary. It can be seen that the curves are almost the same, regardless of the location of the indents within the polycrystalline structure.

\section{Results from the exponential-load method}

Fourteen indentation tests were conducted in the interior of some grains on the $\mathrm{Ni}_{3} \mathrm{Al}$ sample using an exponential loading schedule with a Berkovich indenter. The loading schedule here is composed of an exponential ramp with a time constant of $0.01 \mathrm{~s}^{-1}$ to a maximum force of $8 \mathrm{mN}$ and is superimposed upon with seven sinusoidal modulations each for duration of about $31 \mathrm{~s}$. Each modulation included five cycles of oscillation at $0.25 \mathrm{~Hz}$ with amplitude of $500 \mu \mathrm{N}$.

The indents made in this set of tests were much smaller $(a=0.58 \pm 0.01 \mu \mathrm{m})$ than those made in the tests above due to the small applied load, so that the extent of potential grain boundary influence on hardness is reduced to approximately $3.5 \mu \mathrm{m}$ measured from the center of indent to the closest grain boundary. The contact stiffness was obtained by dividing the amplitude of the load by the amplitude of the displacement with the consideration of phase difference between the two sinusoidal components. ${ }^{17}$ The hardness was then evaluated from the contact stiffness $S$, indentation load $P$, and reduced modulus $E_{\mathrm{r}}$ as $^{11}$

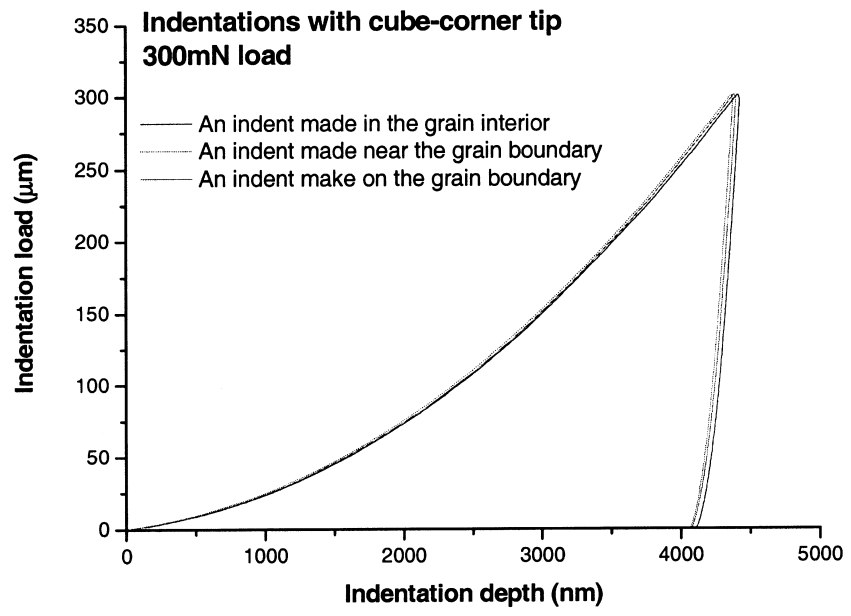

FIG. 4. Typical load-displacement curves of cube-corner indentations made with a maximum load of $300 \mathrm{mN}$ on the grain interior, near a grain boundary, and right on a grain boundary.

$$
H=\frac{4 P E_{\mathrm{r}}^{2}}{\pi S^{2}}
$$

The reduced modulus $E_{\mathrm{r}}$ was computed from the known elastic moduli of $\mathrm{Ni}_{3} \mathrm{Al}\left(C_{11}=230, C_{12}=150\right.$, and $\left.C_{44}=131\right)^{21}$ using the method of Vlassak and $\mathrm{Nix}^{22}$ and the reduced modulus of the diamond tip (1140 GPa) supplied by the manufacturer.

Figure 5 shows the variations of hardness calculated from Eq. (2) during a set of exponential-load indentation experiments performed in the grain interior and also near a grain boundary. It is observed that the hardness value settles down to a steady value at a load of around $5000 \mu \mathrm{N}$. The average steady-state hardness obtained from the 14 indentations in the grain interior was $5.78 \mathrm{GPa}$, with a standard deviation of $0.54 \mathrm{GPa}$. The steady-state hardness value evaluated with Eq. (2) for the indentation made near a grain boundary $(0.18 \mu \mathrm{m}$ from it) was $5.08 \mathrm{GPa}$. This $H$ value is in fact $0.35 \mathrm{GPa}$ lower than one standard deviation from the bulk average hardness and hence does not support the presence of a grain boundary hardening effect.

\section{Summary}

Two conclusions can be drawn from the above observations on the hardness profiles near the grain boundaries. First, results from both statistical hypothesis testing and direct SEM imaging on the indent shapes do not support a rising trend of hardness as the distance from the grain boundary decreases; that is, do not support a grain boundary hardening effect, regardless of the geometry of indenter used or the indentation schedule or load used. Second, the main source of error for $H$ is due to the measurement of indent area from the corresponding SEM image and is within the range of 0.7 to $1.0 \mathrm{GPa}$ at a load of $300 \mathrm{mN}$. This magnitude of measurement error is

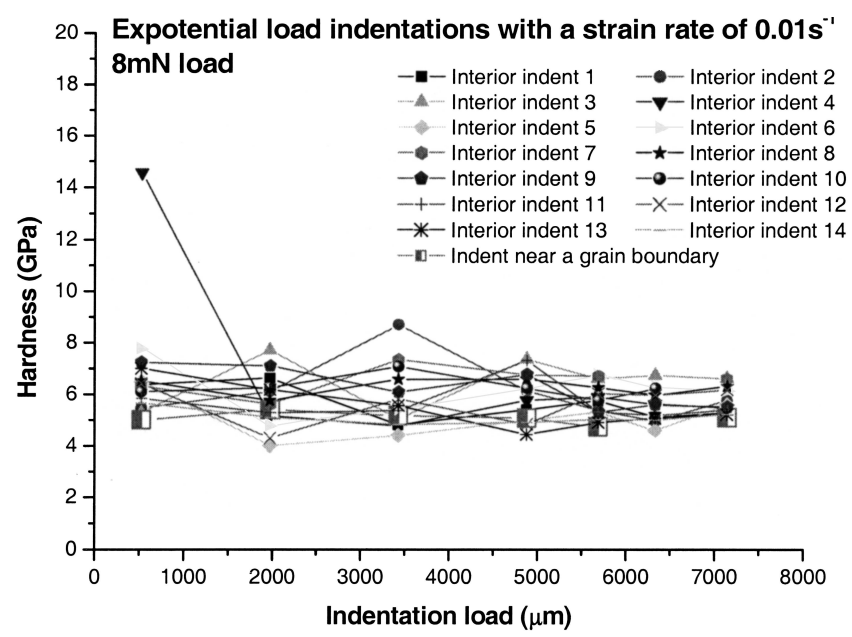

FIG. 5. Hardness against indentation load for indentations using the exponential-load method. 
larger than any deviation of the $H$ value from the bulk mean value encountered at the grain boundary regions. It is also possible that different orientations of the grains may result in slight differences in the measured hardness values.

\section{Indent topography near grain boundaries}

Figure 6 shows the AFM images of two Berkovich indents [Figs. 6(a, i and ii)] and two cube-corner indents [Figs. 6(b, i and ii)] on the $\mathrm{Ni}_{3} \mathrm{Al}$ sample, together with the topographic profiles across the indents along a direction normal to the grain boundary if one is present nearby. The Berkovich indent shown in Fig. 6(a, i) is close to a grain boundary, whereas that shown in Fig. 6(a, ii) is in the interior of the same grain. Similarly, the cube-corner indent in Fig. 6(b, i) is located near a grain boundary and that shown in Fig. 6(b, ii) is in the interior of the same grain. It can be seen that the Berkovich indents, both close to the grain boundary [Fig. $6(\mathrm{a}, \mathrm{i})]$ and in the grain interior [Fig. 6(a, ii)], seem to be identical in terms of the indent shape as well as dimensions. Also, the indent close to a grain boundary remains symmetrical. Similar observations can be made from the cube-corner indent near the grain boundary [Fig. 6(b, i)] and that far from the grain boundary [Fig. 6(b, ii)]. Because hardness is defined as load divided by the projected indent area, a higher hardness corresponds to a smaller projected area under the same load. Also, if an observed increase in hardness is due to difficulty in slip transmission across a grain boundary, distortions in the indent shape should occur near the grain boundary. However, both size reduction or shape distortion are not observed in the indents made near a grain boundary in Fig. 6. The conclusion here is therefore the same as that drawn from the hardness profiles [Figs. 1(a)-1(c)]; namely, that a grain boundary does not exert any obvious effect on the measured hardness in our $\mathrm{Ni}_{3} \mathrm{Al}$ sample.

The above conclusion, however, does not imply that there can be no effect by a grain boundary on other aspects of the indentation apart from the hardness and the indent shape. The observations so far only indicate that the parameter of hardness itself is not sensitive enough to represent the effects of a grain boundary. In fact, it can be observed that when an indent is made very close to a grain boundary, the slip trace patterns between the grain boundary and the indent appear to be distinctively different from those around an indent made under identical conditions in the grain interior. Also, in situations when the indent is so close to a grain boundary that the slip trace zone surrounding the indent sweeps across the grain boundary, discontinuities in the slip trace patterns can be seen at the grain boundary and, in some cases, an extra amount of material pile-up appears in the region between the indent and the grain boundary. Figures $6(\mathrm{a}, \mathrm{i}$ and ii) show the topographic profiles of a Berkovich indent made near a grain boundary and one made within the interior of the same grain using the same load of $300 \mathrm{mN}$. It can be seen that, compared to the indent in Fig. 6(a, ii), an extra amount of pile-up of about $0.05 \mu \mathrm{m}$ in height occurred in the region between the grain boundary and the indent in Fig. 6(a, i). A similar observation can also be made in the cube-corner indents in Figs. 6(b, i and ii), except that the height of the material pile-up around the indent next to the grain boundary in Fig. 6(b, i) is now $0.1 \mu \mathrm{m}$ higher than that of the indent in the interior of the same grain in Fig. 6(b, ii).

Different grain boundaries also exhibit a different degree of intergranular slip transmission, as illustrated in Figs. 7(a)-7(c). At some grain boundaries, slip is largely blocked at the grain boundaries, as shown in Fig. 7(a). Here, clear slip traces cannot be seen in the adjacent grain even though the indent is made very near to the grain boundary, and a significant degree of material pileup can be observed at the grain boundary, leaving what appears to be a step or a crack along the grain boundary. This type of behavior is denoted as the type- $\mathrm{B}$ behavior and is thought to be due to the blockage of slip by the grain boundary, leading to relaxation of strain through pile-up or even localized cracking at the grain boundary. At other grain boundaries, slip can be seen to transmit smoothly to the adjacent grain without much apparent influence by the grain boundary [Figs. 7(b) and 7(c)]. In some cases like that shown in Fig. 7(b), the slip trace zone has clearly extended to the adjacent grain, but steps can also be seen at the grain boundary, suggesting a certain extent of material pile-up. In other cases like Fig. 7(c), the grain boundary does not seem to result in any discontinuity in the shape of the slip trace zone, except for a reorientation of the slip traces in the adjacent grain, which must naturally occur due to the different orientations of the slip planes in the adjacent grain. Also, no clear step or pile-up can be observed at the grain boundary. We describe the behavior in Fig. 7(b) as the intermediate behavior, denoted as type I, and that in Fig. 7(c) as the obvious transmission behavior, denoted as type $\mathrm{T}$.

It is believed that the difference in slip transmission behavior across grains is related to the misorientation between the grain-pair in question. The electron backscattering patterns (EBSPs) in Figs. 7(a)-7(c) in fact illustrate this. In the type-T behavior in Fig. 7(c), the EBSPs on either side of the grain boundary show only slight misorientation: The zone center at the bottom edge of the EBSP of the indented grain is only slightly rotated away from the same zone center of the neighboring grain about a horizontal axis, and there is no rotation about the normal axis of the EBSPs. In the type-I behavior in Fig. 7(b), the two grains are rotated slightly about the horizontal axis as well as the normal axis of the EBSPs. In the type-B behavior in Fig. 7(a), the zone center in the 


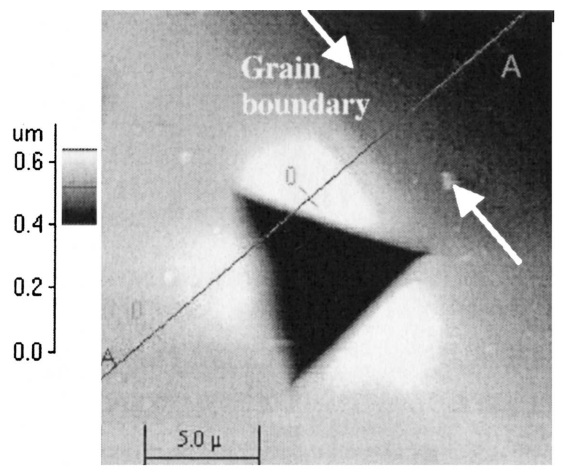

(a)(i)

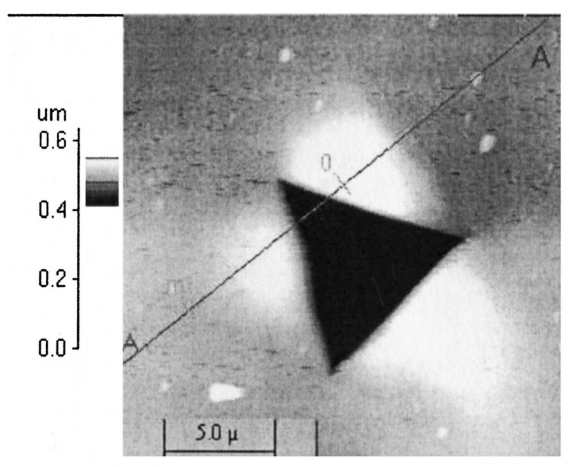

(a)(ii)

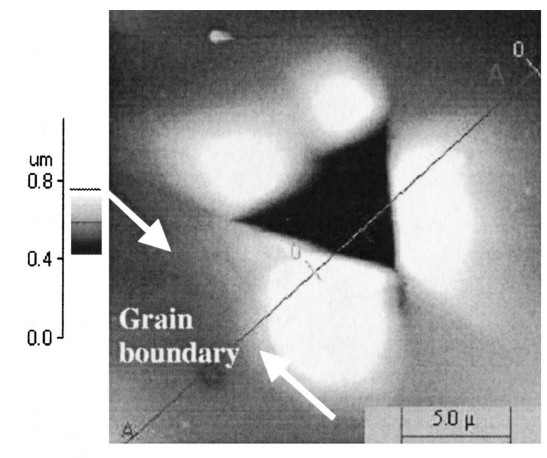

(b)(i)

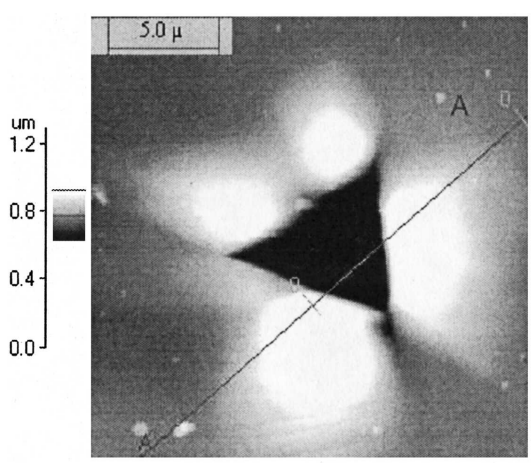

(b)(ii)
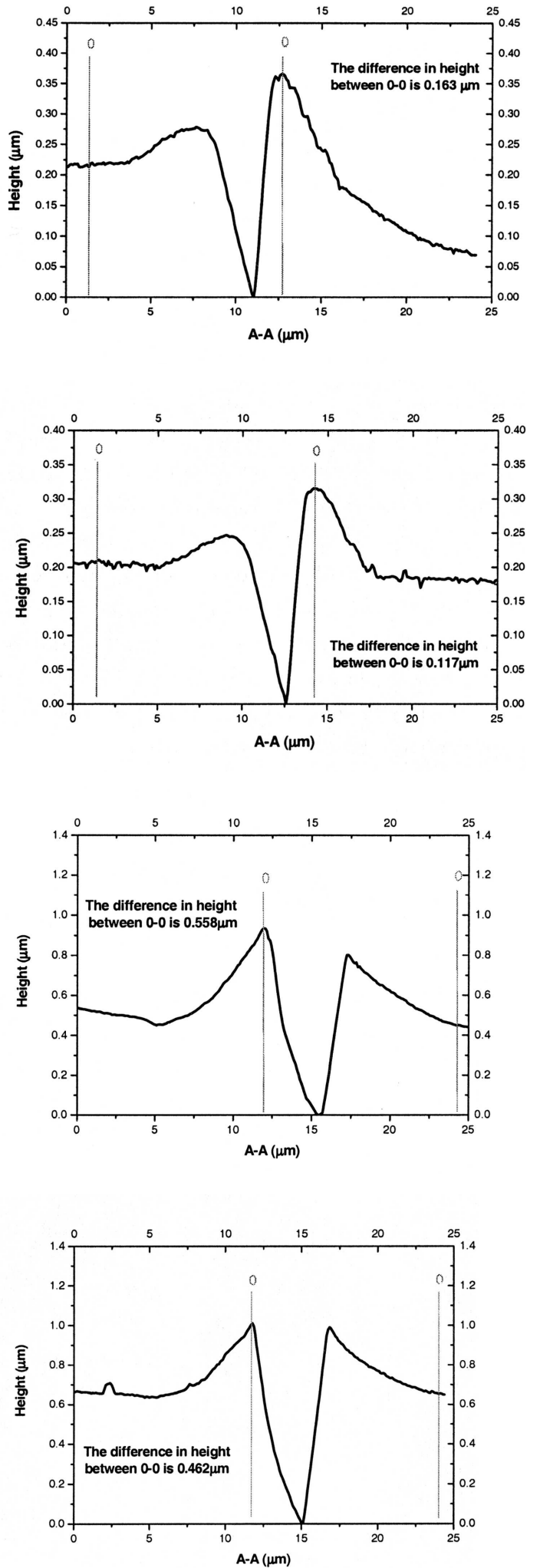

FIG. 6. Non-contact-mode AFM images of some typical (a) Berkovich and (b) cube-corner indents. Next to the images are shown the corresponding topographic profiles across the indent along a direction normal to the grain boundary of interest. $(a, i)$ and $(b, i)$ are indentations near grain boundaries. (a, ii) and (b, ii) are indentations in grain interiors. 


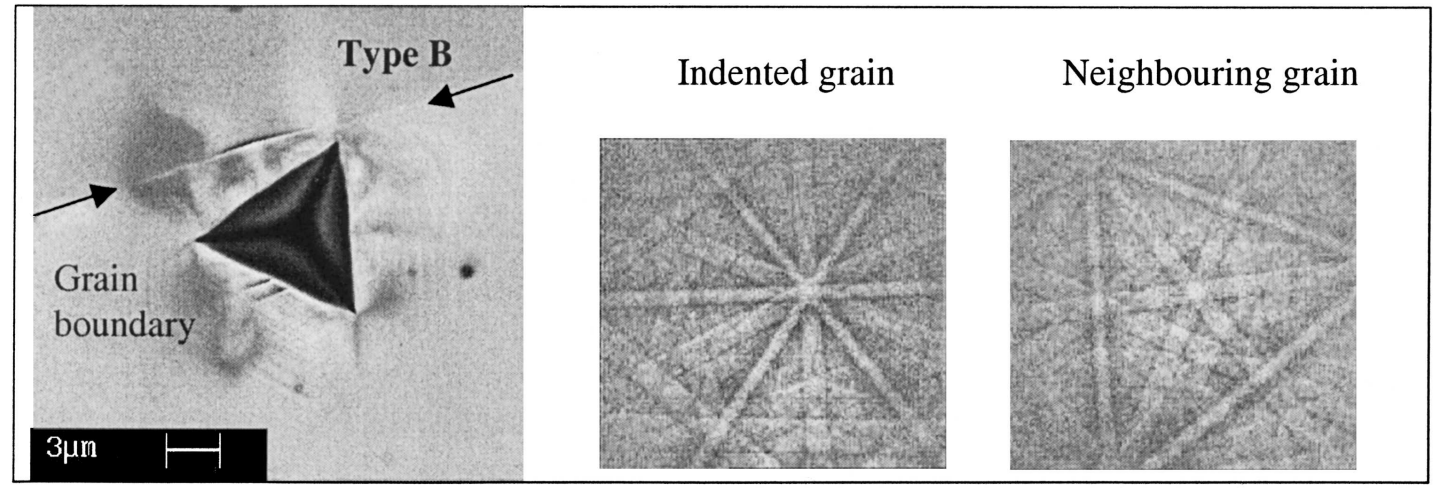

(a)

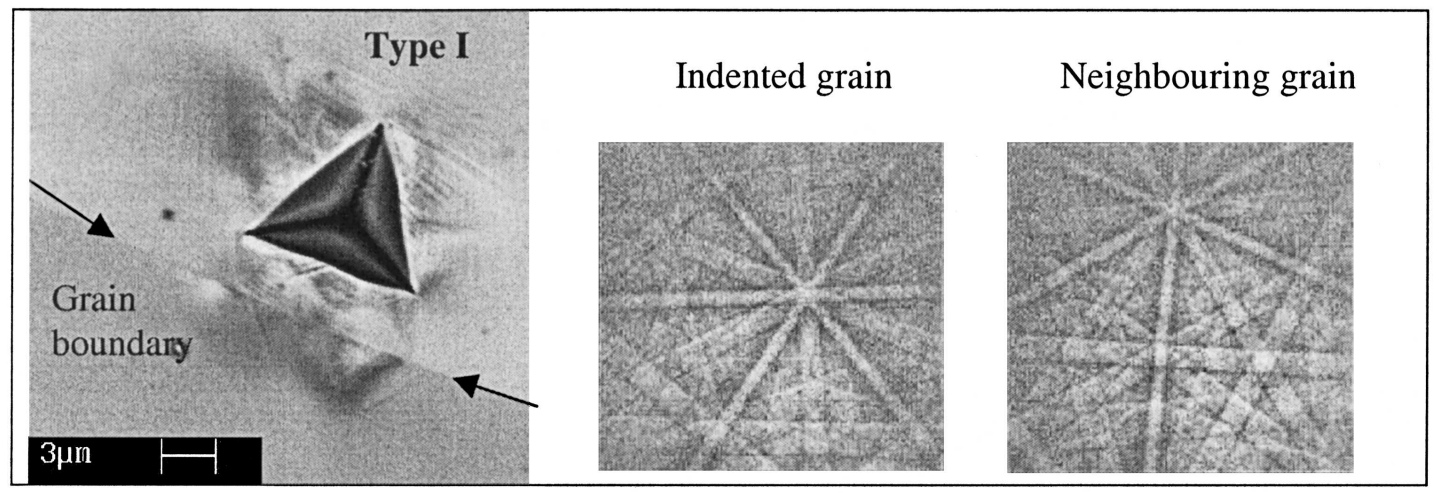

(b)

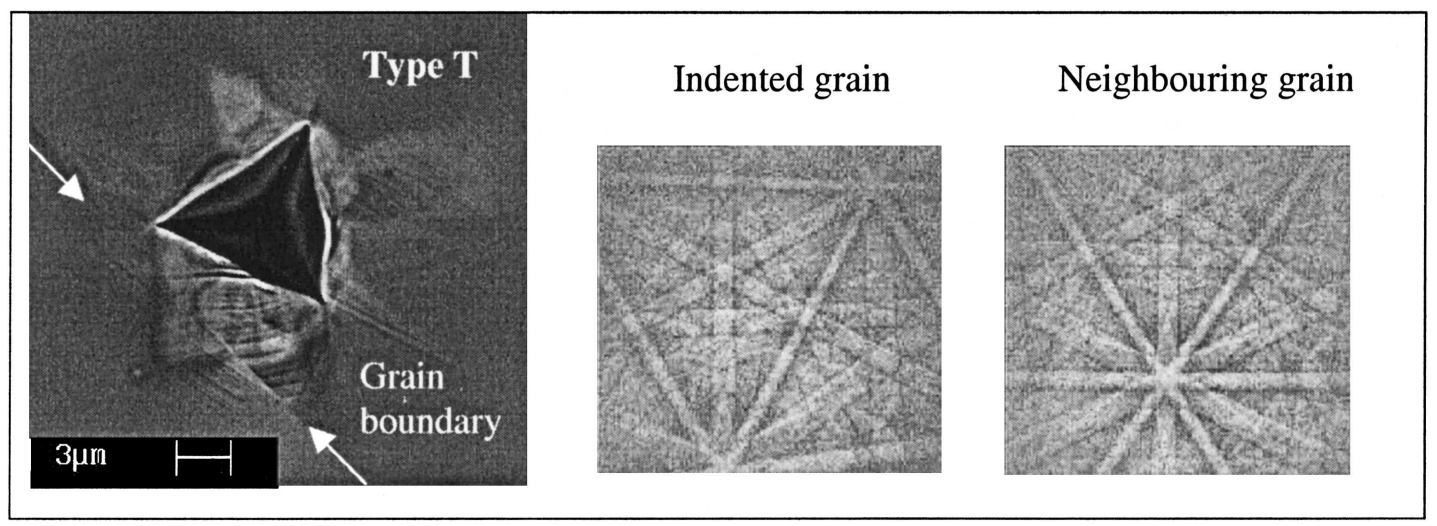

(c)

FIG. 7. SEM backscattered images of three indents made less than $6 \mu \mathrm{m}$ from different grain boundaries. Next to the images are shown the corresponding EBSP of the indented grain and the adjacent grain.

indented grain has shifted significantly away in the neighboring grain, indicating a large misorientation.

To quantify the misorientation effect, a systematic study was carried out to determine the relationship between the extent of intergranular slip transmission and the misorientation between the corresponding grains. As can be seen by comparing Figs. 2(a) and 2(b), a cubecorner tip can produce a larger and more obvious slip trace zone around the indent than a Berkovich indenter under the same load. For this reason, the cube-corner indenter was used for this study. Figures 8(a) and 8(b) show the locations of some of the cube-corner indents relative to the nearby grain boundaries selected to be investigated on the sample. The indents were made along the selected grain boundaries at a separation of about 20 $\mu \mathrm{m}$ to avoid any potential work-hardening effects caused by the previous indents. The misorientations across the selected grain boundaries were measured from the EBSPs on either side of the grain boundaries, as exemplified in Fig. 7. The ease of intergranular slip transmission is compared with respect to a misorientation factor $m^{\prime}$ defined as 


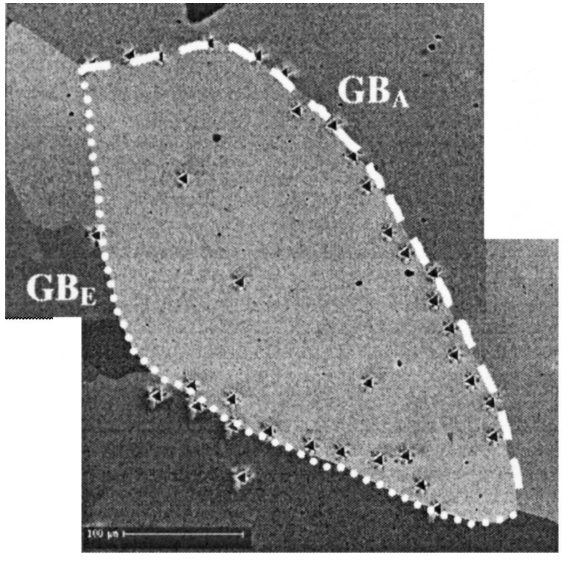

(a)

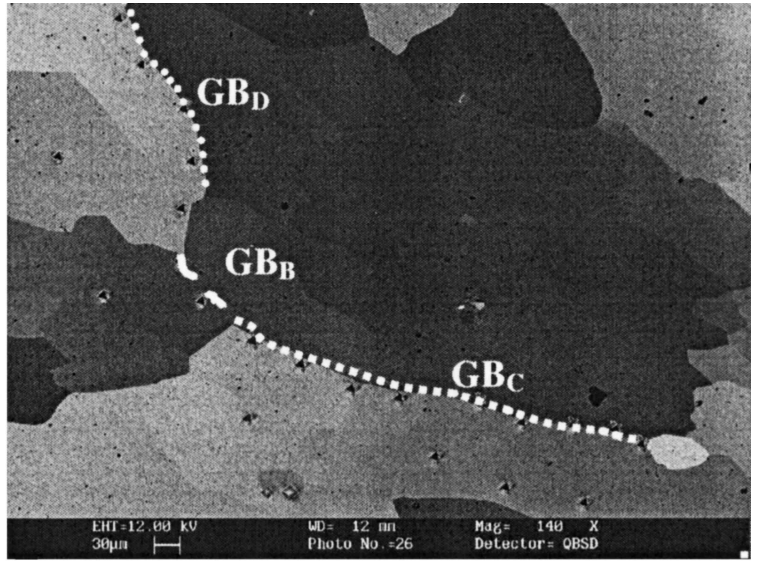

(b)

FIG. 8. SEM backscattered images showing the locations of some of the cube-corner indents made with $300 \mathrm{mN}$ relative to some selected grain boundaries on the $\mathrm{Ni}_{3} \mathrm{Al}$ sample. The grain boundaries indicated in (a) and (b) are referred to in Table I.

$$
m^{\prime}=\cos \theta_{A} \cdot \cos \theta_{B},
$$

where $\theta_{\mathrm{A}}$ is the angular difference of the closest $\{111\}$ slip planes in the two neighboring grains and $\theta_{\mathrm{B}}$ is the angular difference of the closest $\langle 110\rangle$ slip directions on these two slip planes. The slip planes and directions chosen here are appropriate for $\mathrm{Ni}_{3} \mathrm{Al}$ at room temperature.

The slip behavior across different grain boundaries with the corresponding $m^{\prime}$ observed in the sample are summarized in Table I. Also shown are the disorientation of the grain boundaries expressed in the usual misorientation angle/axis notation in the form of 0/UVW as well as the coincident size lattice (CSL) if one can be found. It can be seen that a good correlation exists between the value of $m^{\prime}$ and the ease of intergranular slip transmission; namely, type-B transmission behavior is associated with low $m^{\prime}$ values and type-T with high $m^{\prime}$ values. However, within the sample of grain boundaries studied, there is apparently no simple correlation between the slip transfer behavior and the CSL, as can be seen from the observation that two type- $\mathrm{B}$ grain boundaries $\left(\mathrm{GB}_{\mathrm{C}}\right.$, $\mathrm{GB}_{\mathrm{D}}$ ) are close to CSL $\Sigma 5$ or $\Sigma 29 \mathrm{~b}$, but two other type-B grain boundaries $\left(\mathrm{GB}_{\mathrm{A}}, \mathrm{GB}_{\mathrm{B}}\right)$ are far away from any simple CSL.

\section{DISCUSSION}

The hardness measurements in this work clearly indicate that hardness measurement is not an effective method to probe the slip transfer properties of grain boundaries in our $\mathrm{Ni}_{3} \mathrm{Al}$ sample. This conclusion is in sharp contrast with that reached by Lee et al. ${ }^{9}$ who have also investigated boron-free $\mathrm{Ni}_{3} \mathrm{Al}$ but have concluded a grain-boundary hardening effect and interpreted it in terms of intergranular slip transmission. As mentioned above, the observed hardening phenomenon reported by Lee et al. may in fact be an artefact due to the measurement errors of the indent sizes. The error magnitude of \pm HV 3 quoted by Lee et al. is impossibly small for the type of microindentation equipment used in their study; our experience with a similar microindenter on the same material using the same force leads to an estimated error of $\pm \mathrm{HV}$ 72. Lee et al.'s reported rising trend of hardness from HV 190 to 235 in the grain boundary region (i.e., a variation of HV 45) is indeed well within our estimated error of \pm HV 72 . In other words, through a more realistic estimation of the experimental error, we find the conclusion of Lee et al. on the increase of hardness near a grain boundary very doubtful.

TABLE I. Summary on the orientation factor and the deformation behavior at different grain boundaries on the $\mathrm{Ni}_{3} \mathrm{Al}$ sample.

\begin{tabular}{|c|c|c|c|c|c|c|}
\hline $\begin{array}{l}\text { Related } \\
\text { grains }\end{array}$ & $\cos \left(\theta_{\mathrm{A}}\right)$ & $\cos \left(\theta_{\mathrm{B}}\right)$ & $m^{\prime}$ & $\begin{array}{l}\text { Disorientation } \\
\text { } / \mathrm{UVW}\end{array}$ & $\begin{array}{l}\text { Coincident } \\
\text { size lattice }\end{array}$ & $\begin{array}{l}\text { Deformation behavior } \\
\text { at grain boundary (Fig. 7) }\end{array}$ \\
\hline $\mathrm{GB}_{\mathrm{A}}$ & 0.9102845 & 0.8860473 & 0.81 & $44.2 \% 11-1-4$ & $\cdots$ & Type B \\
\hline $\mathrm{GB}_{\mathrm{B}}$ & 0.8941368 & 0.8775367 & 0.78 & $38.7^{\circ} / 1-40$ & $\cdots$ & Type B \\
\hline $\mathrm{GB}_{\mathrm{C}}$ & 0.8498666 & 0.8879597 & 0.75 & $40.4^{\circ} / 100$ & Near $\sum 5$ and $\sum 29 b$ & Type B \\
\hline $\mathrm{GB}_{\mathrm{D}}$ & 0.8958527 & 0.8517548 & 0.76 & $40.5^{\circ} / 100$ & Near $\sum 5$ and $\sum 29 b$ & Type B \\
\hline $\mathrm{GB}_{\mathrm{E}}$ & 0.9556497 & 0.9327741 & 0.89 & $33.6 \% 1-4-1$ & $\cdots$ & Type I \\
\hline $\mathrm{GB}_{\mathrm{F}}$ & 0.9918736 & 0.9667900 & 0.96 & $17.1^{\circ} / 4-41$ & $\cdots$ & Type I \\
\hline $\mathrm{GB}_{\mathrm{G}}$ & 0.9970575 & 0.9907858 & 0.99 & $17.0 \%-12-1$ & $\cdots$ & Type T \\
\hline $\mathrm{GB}_{\mathrm{H}}$ & 0.9960494 & 0.9922302 & 0.99 & $30.4 \%-14-2$ & $\cdots$ & Type T \\
\hline
\end{tabular}


Apart from the statistical error due to the judgment of the indent size discussed above, the measurement of microhardness using the filar micrometer method is also subject to systematic errors, as discussed in Sec. III. B. We found that measurement of indent sizes using the filar micrometer in our microindenter yields significant overestimation of lengths within the $0-30 \mu \mathrm{m}$ range, leading to underestimation of the Vickers hardness at HV 297.0 \pm 71.5 as opposed to the HV $443.0 \pm 74.6$ obtained by direct SEM imaging of the indents. The measurements of Lee et al. were made on a similar microindenter and may therefore suffer from the same problem. In fact, the hardness range of HV 190 to 235 reported by Lee et al. is only slightly lower than the HV $297.0 \pm 71.5$ (the erroneous value) in the current work. The small discrepancy here may be due to the different levels of sample impurities in the two studies as well as to the different interpretation of indent size as seen in the optical microscope of the microindenter (i.e., a systematic judgmental error).

Another possibility may be that a hardening effect indeed exists in the sample of Lee et al., but this is due to solid-solution hardening as a result of impurity segregation at the grain boundary. It should be noted that the sample used by Lee et al. was a bicrystal rather than a polycrystalline sample as in the current work. Because the grain boundary density of a bicrystal could be orders of magnitude lower than that of a polycrystalline specimen, it is highly possible that grain-boundary segregation of impurities could be much more severe in a bicrystal than in a polycrystalline sample. If this is the reason for a grain-boundary hardening effect in the case of Lee et al., their interpretation in terms of intergranular slip transmission would be invalid. Another problem with Lee et al.'s interpretation of their results is that they observed the highest hardness to occur when the indent is made right on the grain boundary. If their interpretation of the hardening being due to difficulties in intergranular slip transmission is correct, then the hardness measured right on the grain boundary should be free from such an effect, as now both grains on either side of the grain boundary can start deformation simultaneously as the indent now covers both grains. One would therefore expect the hardness value to be comparable or even lower than that within the grain interior.

By analyzing the data of Lee et al. on $\mathrm{Ni}_{3} \mathrm{Al}$, Ngan and $\mathrm{Chiu}^{7}$ have concluded an apparent Hall-Petch relationship between the hardness and the distance from a grain boundary. On the basis of the current experimental results and the reanalysis of Lee et al.'s data above, we have to conclude here that the apparent Hall-Petch relationship in $\mathrm{Ni}_{3} \mathrm{Al}$ reported by $\mathrm{Ngan}$ and Chiu is fortuitous if not erroneous and should not serve as supporting evidence for an interpretation of a hardening effect by the difficulty of intergranular slip transmission in this material. Ngan and Chiu have also found similar Hall-Petch relationships for other materials using existing hardness data from the literature, but in the absence of careful experimental results on each of these materials, it would be inappropriate here to draw definite conclusions on the effect of slip transmission across grain boundaries on the hardness values in these materials.

The idea of grain-boundary hardening effect due to slip transmission difficulties across grain boundaries was also considered in a recent study by Soifer et al. ${ }^{23}$ in which nanoindentation was performed on high-purity polycrystalline copper to probe the strength of individual grain boundaries. It was found that, under a load of $90 \mu \mathrm{N}$, both hardening or softening occurred on adjacent grains as the distance from the grain boundary decreased. Though Soifer et al. did not reject the possibility of grain boundary hardening being due to slip transmission difficulties across the grain boundary, they found this reason questionable, as the difficulty of slip transmission alone cannot explain the coexistence of grain boundary softening and hardening in adjacent grains. It should also be noted that the indentation loads used by Soifer et al. were three orders of magnitude smaller than the loads used in the current work, and their indents were several hundreds of nanometers in size instead of several micrometers as in this work. We used large indentation loads in this work because we wanted to ensure that the indents were deep enough compared to any trenches or surface steps that might arise due to preferential etching effects at the grain boundaries. The typical topographic profiles in Figs. 6(a, i) and $6(b, i)$ confirm this expectation. If the indents are too shallow, apart from possible influences by the etching steps or trenches as discussed by Soifer et al., the hardness may also be subjected to effects of surface oxide layers. ${ }^{24}$

The current results in Fig. 7 and Table I show that a grain boundary can exert visible effects on the appearance of the slip traces around an indent. Such effects may of course be interpreted in terms of the ability of the grain boundary to transmit slip. In the widely accepted mechanism to explain the Hall-Petch effect, ${ }^{25}$ the Hall-Petch slope $k_{y}$ is interpreted as $m m^{\prime} \tau_{c} r_{c}^{1 / 2}$ where $\tau_{\mathrm{c}}$ is the dislocation source strength, $r_{\mathrm{c}}$ is the typical distance of a dislocation source from a grain boundary, $m$ is the macroscopic orientation factor relating tensile stress to shear stress in a polycrystalline specimen, and $m^{\prime}$ is a local misorientation factor between slip systems across a grain boundary. In a bicrystal-like experiment as in the case of subgranular indentation, the ease of slip transmission should thus be characterized by the factor $m^{\prime}$ alone, which is defined in Eq. (3). According to the results in Table I, it can be observed that the ease of intergranular slip transmission in the $\mathrm{Ni}_{3} \mathrm{Al}$ sample seems to exhibit a strong correlation with the value of $m^{\prime}$. It can be seen that when $m^{\prime}$ is smaller than approximately 0.81 , the slip transmission exhibits the type-B behavior in Fig. 7(a), in 
which slip is largely blocked by the grain boundary, and with material piling up or cracking at the grain boundary. When $m^{\prime}$ increases beyond approximately 0.89 , the slip transmission behavior becomes type I in Fig. 7(b) or even type $\mathrm{T}$ in Fig. 7(c). The observed trend here for $\mathrm{Ni}_{3} \mathrm{Al}$ therefore supports the idea that the slip transmission ability of a grain boundary is mainly governed by the misorientation between slip planes and slip directions across the grain boundary. This result agrees with the fundamental assumption in the physical explanation of the Hall-Petch effect, which has never been properly verified up to this date.

Finally, as noted above, the results in Table I seem to indicate that, within the sample of grain boundaries studied here, the slip transmission across CSL boundaries does not appear to be easier or more difficult than across general boundaries. On the other hand, special grain boundaries are known to produce extrinsic grain boundary dislocations that can exert localized effects on slip transmission. ${ }^{26-31}$ It should be noted that the current experiments did not involve a large enough collection of special boundaries to systematically investigate their localized effects. Future experiments can be performed to study the effects of special boundaries.

\section{CONCLUSIONS}

Repeated experimentation using different techniques of micro- and nanoindentation shows that hardness measurement is an ineffective means to probe the slip transmission ability of a grain boundary in $\mathrm{Ni}_{3} \mathrm{Al}$. The slip trace pattern around an indent, however, is sensitive to the presence of a grain boundary. It was found that the observed ease of slip transmission is highly correlated to the misorientation of slip planes and slip vectors between the adjacent grains.

\section{ACKNOWLEDGMENT}

The work described in this paper was supported by a grant from the Research Grants Council of the Hong Kong Special Administrative Region, People's Republic of China (Project No. HKU 7062/01E).

\section{REFERENCES}

1. K.T. Aust, R.E. Hanneman, P. Niessen, and J.H. Westbrook, Acta Metall. 16, 291 (1968).

2. T. Watanabe, S. Kitamura, and S. Karashima, Acta Metall. 28, 455 (1980)

3. L.B. Harris, V.R. Howes, and N.G. Cutmore, J. Am. Ceram. Soc. 65, 35 (1982).

4. Y.T. Chou, B.C. Cai, A.D. Romig, Jr., and L.S. Lin, Philos. Mag. A 47, 363 (1983).

5. Z.Q. Zhou and Y.T. Chou, J. Less-Common Met. 114, 323 (1985).

6. J.W. Wyrzykowski and M.W. Grabski, Philos. Mag. A. 53, 505 (1986).

7. A.H.W. Ngan and Y.L. Chiu, in Fundamentals of Nanoindentation and Nanotribology II, edited by S.P. Baker, R.F. Cook, S.G. Corcoran, and N.R. Moody (Mater. Res. Soc. Symp. Proc. 649, Warrendale, PA, 2001), p. Q4.10.

8. E.M. Schulson, T.P. Weihs, I. Baker, H.J. Frost, and J.A. Horton, Acta Metall. 34, 1395 (1986).

9. C.S. Lee, G.W. Han, R.E. Smallman, D. Feng, and J.K.L. Lai, Acta Mater. 47, 1823 (1999).

10. B.N. Lucas and W.C. Oliver, Metall. Mater. Trans. A 30A, 601 (1999).

11. S.A. Syed Asif and J.B. Pethica, Philos. Mag. A. 76, 1105 (1997).

12. L.E. Samuels and T.O. Mulhearn, J. Mech. Phys. 5, 125 (1956).

13. K.L. Johnson, J. Mech. Phys. Solids 18, 115 (1970).

14. D. Tabor, The Hardness of Metals (Clarendon Press. Oxford, U.K., 1951), pp. 160.

15. W. Wang and K. Lu, J. Mater. Res. 17, 2314 (2002).

16. W.D. Nix and H.J. Gao, Mech. Phys. Solids 46, 411 (1998).

17. W.C. Oliver and G.M. Pharr, J. Mater. Res. 7, 1564 (1992).

18. G. Feng and A.H.W. Ngan, J. Mater. Res. 17, 660 (2002).

19. A.H.W. Ngan and B. Tang, J. Mater. Res. 17, 2604 (2002).

20. B. Tang and A.H.W. Ngan, J. Mater. Res. 18, 1141 (2003).

21. A.F. Voter and S.P. Chen, in Characterization of Defects in Materials, edited by R.W. Siegel, J.R. Weertman, and R. Sinclair (Mater. Res. Soc. Symp. Proc. 82, Pittsburgh, PA, 1987), p. 175.

22. J.J. Vlassak and W.D. Nix, J. Mech. Phys. Solids 42, 1223 (1994).

23. Y.M. Soifer, A. Verdyan, M. Kazakevich, and E. Rabkin, Scr. Mater. 47, 799 (2002).

24. Y. Liu and A.H.W. Ngan, Scr. Mater. 44, 237 (2001).

25. R.E. Smallman and R.J. Bishop, Metals and Materials: Science, Processes, Applications (Butterworth-Heinemann, Oxford, U.K., 1995), pp. 229.

26. W. Bollmann, Crystal Defects and Crystalline Interfaces (Springer-Verlag, Berlin, 1970).

27. H. Gleiter and P. Pumphrey, Mater. Sci. Eng. 25, 159 (1976).

28. T. Malis and K. Tangri, Acta Metall. 27, 25 (1979).

29. Z. Shen, R.H. Wagoner, and W. Clark, Acta Metall. 36, 3231 (1988).

30. S. Sangal, K.J. Kurzydlowski, and K. Tangri, Acta Metall. Mater. 39, 1281 (1991).

31. L.H. Friedman and D.C. Chrzan, Philos. Mag. A 77, 1185 (1998). 